\title{
Energy Density of Gravitational Field in General Transverse Gauge
}

\author{
Ben-Chao Zhu \\ Department of physics and mathematics, Hubei \\ University of Medicine, shiyan, China \\ Guang-Xiao He \\ Department of physics and mathematics, Hubei \\ University of Medcine, shiyan, China
}

\author{
Jia Guo \\ Department of physics and mathematics, Hubei \\ University of Medicine, shiyan, China \\ Peng-Cheng Zhang* \\ Department of physics and mathematics, Hubei \\ University of Medicine, shiyan, China \\ e-mail: pengchengzhang@163.com \\ */corresponding author
}

\begin{abstract}
In this paper, firstly, we will prove there is a general transverse gauge in general relativity for linear approximation. This gauge condition can play the same role as the radiation gauge condition in electrodynamics. It means that the non-physical metric tensor and affine connection can be removed by this general transverse gauge. Then, by using this gauge condition, the energy density of gravitational can be revisited. we prove that the energy distribution of pure gravitational field out of the source can be upgraded by using the Einstein, Weinberg and LandauLifshitz energy-momentum pseudo-tensors in general transverse gauge. Furthermore, the different parameter in general transverse gauge will bring some interesting conclusions, and these different results are also discussed detail in this paper. Finally, by the concrete computing the energy density of one gravitational source, we find the expression for Einstein is the most useful definition of energy-momentum in calculating energy density of gravity. This result successfully overcomes the last-standing problem of energy density of gravitational field .
\end{abstract}

Keywords- general relativity; transvers gauge;energymomentum; pseudo-tensors; energy density

\section{INTRODUCTION}

Reality is described by modern physical theories in terms of fields, e.g., the electromagnetic field, the gravitational field, and fields for the electron and all other elementary particles. Remarkably, all these fields theories can be built by a so-called gauge invariance principle which is imposed on the Lagrangian of fields. Unfortunately, the term gauge refers to redundant degrees of freedom in the Lagrangian should be got ride of when identifying the real physical degrees of freedom, although these redundant field components can help to write down an elegant Lagrangian. Thus finding a pertinent gauge fixing to remove all gauge freedoms is always a meaningful work in gauge theories. The actual situation is that one uses a four-dimensional vector field $A^{\mu}$ to describe a massless photon with two physical polarizations in electrodynamics. Radiation (or Coulomb, transverse) gauge fixing $\stackrel{v}{\nabla} \cdot \stackrel{A}{A}=0$ can perfectly remove the gauge freedom completely and specify the transverse field $A_{\perp}$ to be two physical components which propagate in electromagnetic radiation [1]. When handling gravitational field, the situation becomes troublesome as one uses a tencomponents symmetric tensor $\mathrm{g}_{\mu v}$ to describe gravitational field, but the number of physical degrees of freedom is still two [2]. So finding a gravitational "radiation gauge "as satisfactory as $\nabla \cdot A=0$ in electrodynamics is much harder. In this paper, we will show that the corresponding gravitational "radiation gauge" in linear approximation is

$$
\partial^{i} h_{i}^{\rho}+b \partial^{\rho} h_{i}^{i}=0 \quad(b \neq-1)
$$

Greek indices run from 0 to 3 , Latin indices run from 1 to 3 , and repeated indices are summed over. Since gravitational "radiation gauge" can play the same role as radiation gauge $\nabla \cdot \stackrel{v}{\nabla}=0$ in electrodynamics, it absolutely can pick out the two pure physical components of gravitational field. One can expect that the energy density of gravitational field constructed by pure physical metric tensor must be gauge-invariant. In order to get gaugeinvariant energy density of gravitational field, we will calculate energy density of a specific gravitational source by using three frequently used energy-momentum pseudotensors of gravitational field, e.g., Einstein's, LandauLifshitz's and Weinberg's [3].

\section{GAUGE FIXING PROBLEM IN ELECTRODYANMICS}

As one uses a four-dimensional vector field $A^{\mu}$ to describe electromagnetic field $\stackrel{v}{E}=-\stackrel{v}{\nabla} A^{0}-\partial_{t} A$ and $\stackrel{\mathrm{v}}{B}=\stackrel{\mathrm{v}}{\nabla} \times \stackrel{\mathrm{v}}{A}$. The field strength, $F_{\mu \nu}=\partial_{\mu} A_{\nu}-\partial_{\nu} A_{\mu}$, is 
invariant under gauge transformation $A_{\mu}^{\prime}=A_{\mu}-\partial_{\mu} \omega$ $(\omega(x)$ is an arbitrary scalar function ). Under this gauge transformation, $F_{\mu \nu}^{\prime}=\partial_{\mu} A_{v}^{\prime}-\partial_{\nu} A_{\mu}^{\prime}=F_{\mu \nu}$ means the field strength tensor is gauge-invariant. The existence of gauge freedom in $A^{\mu}$ shows that one can't expect fixing $A^{\mu}$ only by solving Maxwell equation $\partial_{\mu} F^{\mu v}=-j^{v}\left(j^{\mu}\right.$ is the four-dimensional electric current density)). It means that there must be one additional restriction on vector field, which is called gauge fixing condition. On the one hand, different gauge fixing conditions of vector gauge field will produce different degrees of difficulty on solving Maxwell equation in mathematics. Lorentz gauge condition, $\partial_{\mu} A^{\mu}=0$, is the most frequently useful one. This condition can make Maxwell equation to a simple expression as

$$
\mathrm{W} A^{v}=-j^{v}
$$

$\mathrm{W}=-\partial_{t}^{2}+\nabla^{2}$. Eq. (2) is just the wave equation, and it means all the four components of $A^{\mu}$ are dynamical. But the actual situation is that only two of $A^{\mu}$ are dynamical and physical propagated, so we can conclude that Lorentz gauge can't totally pick out the two pure physical. One the other hand, only radiation gauge, $\nabla \cdot A=0$, can truly remove gauge freedom of $A^{\mu}$. Maxwell equation in radiation gauge reads [4]

$$
\begin{gathered}
\stackrel{\mathrm{v}}{ }^{2} A^{0}=-j^{0} \\
\mathrm{~W} A^{i}-\partial_{t} \partial^{i} A^{0}=-j^{i}
\end{gathered}
$$

$A^{\mu}$ is separated into space and time components. Solving $A^{0}$ by Eq. (3), and it is not propagated by time. By using the conservation condition $\partial_{\mu} j^{\mu}=0$, Eq. (4) can be reduced to

$$
\mathrm{WA}_{\perp}-\stackrel{\mathrm{v}}{j}_{\perp}
$$

We can find only $\mathscr{A}_{\perp}$ is dynamical and has two independent components. $\stackrel{\mathrm{V}}{j_{\perp}}=\stackrel{\mathrm{V}}{j}-\stackrel{\mathrm{V}}{\nabla} \underset{\nabla^{2}}{\stackrel{V}{V}}(\stackrel{\mathrm{V}}{\nabla} \cdot \stackrel{\mathrm{V}}{j})$ is the transverse part of the electric current density.

\section{GENERAL TRANSVERSE GAUGE CONDITON OF GRAVITATIONAL FIELD}

In general relativity, gauge-invariance refers to general covariance under arbitrary coordinate transformation. One usually uses a ten-component symmetric tensor $\mathrm{g}_{\mu v}$ to describe the gravitational field, the number of physical degrees of freedom is the same as massless photon. Obviously, the gauge fixing problem is much harder than that in electrodynamics. When handling gravitational radiation, there is a frequently used gauge named harmonic or De Donder gauge $\mathrm{g}^{\mu v} \Gamma_{\mu v}^{\rho}=0$, which is always regarded as playing the similar role as the Lorentz gauge $\partial_{\mu} A^{\mu}=0$ in electrodynamics. To the sake of simplicity, we will only focus on the gravitational weak-field approximation throughout this paper. Mathematically, gravitational weak-field approximation means one can do the following decomposition about metric tensor: $\mathrm{g}_{\mu v}=\eta_{\mu v}+h_{\mu v} \quad$, with $\eta_{\mu v}=\operatorname{diag}\{-1,1,1,1\} \quad$ is Minkowski metric and $\left|h_{\mu \nu}\right|=1$. It is easy to linearize harmonic gauge as $\partial^{\mu} h_{\mu}^{\rho}-\frac{1}{2} \partial^{\rho} h_{\mu}^{\mu}=0$. Thus the linearized Einstein equation [5]

$$
\mathrm{W} h_{\mu v}-\partial_{\mu} \partial_{\rho} h_{v}^{\rho}-\partial_{\nu} \partial_{\rho} h_{\mu}^{\rho}+\partial_{\mu} \partial_{\nu} h_{\rho}^{\rho}=-S_{\mu v}
$$

can be reduced to the familiar wave equation with source form:

$$
\mathrm{Wh}_{\mu v}=-S_{\mu v}
$$

Here $S_{\mu \nu} \equiv T_{\mu \nu}-\frac{1}{2} \eta_{\mu \nu} T_{\rho}^{\rho}$, and we have put $16 \pi G=1$. From Eq. (7), we can find it is as the same as Eq. (2). It means that it can not fix the gauge completely, and is not sufficient to specify the two physical polarizations of the gravitational wave.

Just as we had presented in the first section of the paper, Eq. (1) is the general transverse gauge condition in general relativity. Firstly, Eq. (1) contains four and only four realizable constraints. Secondly, when we casting Eq. (1) to Eq. (6), we can get

$$
\begin{aligned}
& \stackrel{v}{\nabla} h_{0 \mu}+(1+2 b) \partial_{\mu} \partial_{t} h_{k}^{k}=-S_{0 \mu} \\
& \mathrm{W}_{i j}+\partial_{t}\left(\partial_{i} h_{0 j}+\partial_{j} h_{0 i}\right)+ \\
& (1+2 b) \partial_{i} \partial_{j} h_{k}^{k}-\partial_{i} \partial_{j} h_{0}^{0}=-S_{i j}
\end{aligned}
$$

Combine Eq. (8-9) and Eq. (1) with the conservation condition in the weak-field approximation $\partial_{\mu} T_{v}^{\mu}=0$, we can get the following equations

$$
\begin{gathered}
\nabla^{2} h_{k}^{k}=-\frac{1}{1+b} T_{00} \\
\stackrel{\mathrm{V}}{\nabla}^{2} h_{0 \mu}=-S_{0 \mu}+\frac{1+2 b}{1+b} \frac{1}{\nabla^{2}} \partial_{t} \partial_{\mu} T_{00} \\
\partial_{i} h_{i j}=\frac{b}{1+b} \frac{1}{\nabla^{2}} \partial_{j} T_{00} \\
S_{i j}=S_{i j}+\frac{1}{\nabla^{2}}\left(\partial_{k} \partial_{j} S_{i k}+\partial_{k} \partial_{i} S_{j k}-\partial_{i j} \partial_{j} S_{k k}\right) \\
-\frac{1+2 b}{1+b} \frac{1}{\nabla^{2}} \partial_{i} \partial_{j}\left(S_{k k}-\frac{1}{\nabla^{2}} \partial_{k} \partial_{l} S_{k l}\right)
\end{gathered}
$$

Eqs. (10-13) can remove doubts about why must be $b \neq-1$ in Eq. (1). Eqs. (10-13) are as the same as Eqs. (3$4)$, and we can find only space component of $h_{\mu v}$ are dynamical and propagated. Furthermore, Eq. (14), which plays the same role as $\dot{j}_{\perp}$ in Eq. (5), has two independent degree of freedoms $(6-4=2)$. Because of the parameter $b$, it means that Eq. (1) is not unique, and we call it 
general transverse gauge condition. Thus, we have finish to prove that Eq. (1) is the general transverse gauge condition of gravitational field.

\section{ENERGY DENSITY OF GRAVITATIONAL FIELD IN GENRAL TRANSVERSE GAUGE CONDITION}

Considering a weak, static and spherical source with total mass $M$, a constant mass density $\rho$ and inner pressure $p=0$. We can easily get [6]

$$
\begin{gathered}
T_{\mu \nu}=\operatorname{diag}\{\rho, 0,0,0\} \\
S_{\mu \nu}=\frac{1}{2} \operatorname{diag}\{\rho, \rho, \rho, \rho\}
\end{gathered}
$$

The familiar external solution in the harmonic gauge is

$$
\begin{gathered}
d s^{2}=-\left(1-\frac{2 G M}{r}\right) d t^{2}+\left(1+\frac{2 G M}{r}\right) d x^{2} \\
h_{\mu \nu}=\delta_{\mu \nu} \frac{2 G M}{r}
\end{gathered}
$$

In the general transverse gauge condition, Eq. (1), the external solution of static Schwarzschild solution takes a rather peculiar form as

$$
\begin{aligned}
d s^{2} & =-\left(1-\frac{2 G M}{r}\right) d t^{2}+\left(1+\frac{1-b}{1+b} \frac{G M}{r}\right) d x^{\mathrm{V}_{2}} \\
& -\frac{1+3 b}{1+b} \frac{G M}{r} \frac{(\stackrel{\mathrm{V}}{x} \cdot d \underline{\mathrm{V}})^{2}}{r^{2}} \\
h_{0 m} & =d_{0 m} \frac{2 G M}{r} \\
h_{i j} & =\frac{G M}{r}\left(\frac{1-b}{1+b} d_{i j}+\frac{1+3 b}{1+b} \frac{x_{i} x_{j}}{r^{2}}\right)
\end{aligned}
$$

We will use Eq. (18) and Eq. (20) shortly below to study the energy density of the static Schwarzschild solution.

Once one wants to get the energy density of gravitational field, the first problem is to use which energy-momentum "tensors" (although these tensors are pseudo-tensors). It is useful to shortly review the nonunique problem of energy-momentum in general relativity. In the special theory of relativity, the energy-momentum conservation law of matter plus non-gravitational fields , $\partial_{\mu} T_{v}^{\mu}=0$, are widely accepted. When dealing with the same problem of energy-momentum conservation law in general relativity, it is a straightway to replace the common differential by covariant differential ,e.g. $D_{\mu} T_{v}^{\mu}=0$, and $D_{\mu}=\partial_{\mu}-\Gamma$ is the covariant differential. Unfortunately, the covariant differential can not reduce to $\partial_{\mu} T_{v}^{\mu}=0$ in general case. In order to remove that divergence and obtain the conventional energy-momentum conservation law form. Einstein introduced a quantity $t_{v}^{\mu}$ representing the pure gravitational field energy-momentum tensor, so that the conservation law form can be rewritten as [7]

$$
\partial_{\mu}\left[\sqrt{-g}\left(t_{v}^{\mu}+T_{v}^{\mu}\right)\right]=0
$$

$t_{v}^{\mu}$ is homogeneous quadratic in the first derivatives of the metric tensor and thus it is obviously not a tensor. Unfortunately, Einstein's energy-momentum pseudotensors is not unique. Following Einstein , many scientists like Landau-Lifshitz, MØller, Papapetrou and Weinberg introduced their own energy-momentum., but non of theirs overmatched others completely and the non-tensorial property of energy-momentum was still existed. Since more available energy-momentum tensor definition of gravitational field is absent, many researchers still use those pseudo-tensors to calculate total energy in various space-times. General covariant principle taught us that $t_{v}^{\mu}$ can be made to vanish at a particular point with a suitable choice of a coordinates system. So no one uses these energy-momentum pseudo-tensors to calculate energy of pure gravitational field. This can be certainly attributed to that metric $\mathrm{g}_{\mu \nu}$ has gauge degrees of freedom. Before calculating energy density of gravity, we should declare that the way to get gauge-invariant energy density of gravitational field is not to develop other new energymomentum definition for gravitational field in this paper, but to use pure physical metric tensor, which can be obtained by solving Einstein equation in the radiation gauge, to construct these energy-momentum pseudotensors .

Faced with those too many pseudo-tensors about gravitational field energy-momentum, it is wisely to classify them into different categories before the specific calculations. If we carefully study those energymomentum pseudo-tensors, we can roughly fall those into three categories: (a) Einstein's pseudo-tensor and those developed from Einstein's by using certain surface term; (b) the Landau-Lifshitz (LL) symmetric pseudo-tensor; and (c) the Weinberg pseudo-tensor aimed at covariant property of metric [8]

$$
\begin{aligned}
t_{\mu}^{\tau(\text { Eins })}= & \frac{1}{\sqrt{-g}}\left[\left(g^{\alpha \beta} \sqrt{-g}\right)_{, \mu}\left(\Gamma_{\alpha \beta}^{\tau}-\delta_{\beta}^{\tau} \Gamma_{\alpha \sigma}^{\sigma}\right)\right. \\
& \left.-\delta_{\mu}^{\tau} g^{\alpha \beta}\left(\Gamma_{\alpha \beta}^{\sigma} \Gamma_{\sigma \rho}^{\rho}-\Gamma_{\alpha \sigma}^{\rho} \Gamma_{\beta \rho}^{\sigma}\right) \sqrt{-g}\right] \\
t_{\mu \nu}^{(L L)}= & \frac{1}{2}\left[\left(2 \Gamma_{\alpha \beta}^{\sigma} \Gamma_{\sigma \rho}^{\rho}-\Gamma_{\alpha \rho}^{\sigma} \Gamma_{\sigma \beta}^{\rho}-\Gamma_{\alpha \sigma}^{\sigma} \Gamma_{\beta \rho}^{\rho}\right)\right. \\
& \times\left(g^{\varepsilon \alpha} g^{\tau \beta}-g^{\alpha \beta} g^{\varepsilon \tau}\right) \\
& +g^{\beta \sigma} g^{\varepsilon \alpha}\left(\Gamma_{\alpha \rho}^{\tau} \Gamma_{\beta \sigma}^{\rho}+\Gamma_{\beta \sigma}^{\tau} \Gamma_{\alpha \rho}^{\rho}\right. \\
& \left.-\Gamma_{\sigma \rho}^{\tau} \Gamma_{\alpha \beta}^{\rho}-\Gamma_{\alpha \beta}^{\tau} \Gamma_{\sigma \rho}^{\rho}\right) \\
+ & g^{\beta \sigma} g^{\tau \alpha}\left(\Gamma_{\alpha \rho}^{\varepsilon} \Gamma_{\beta \sigma}^{\rho}+\Gamma_{\beta \sigma}^{\varepsilon} \Gamma_{\alpha \rho}^{\rho}\right. \\
- & \left.\Gamma_{\sigma \rho}^{\varepsilon} \Gamma_{\alpha \beta}^{\rho}-\Gamma_{\alpha \beta}^{\varepsilon} \Gamma_{\sigma \rho}^{\rho}\right) \\
- & \left.g^{\alpha \beta} g^{\rho \sigma}\left(\Gamma_{\alpha \sigma}^{\varepsilon} \Gamma_{\beta \rho}^{\tau}-\Gamma_{\alpha \beta}^{\varepsilon} \Gamma_{\rho \sigma}^{\tau}\right)\right] \\
t_{\mu \nu}^{(W e i n)} & =-\frac{1}{2} h_{\mu \nu} R_{\rho}^{(1) \rho}+\frac{1}{2} \eta_{\mu \nu} h^{\rho \sigma} R_{\rho \sigma}^{(1)} \\
& +R_{\mu \nu}^{(2)}-\frac{1}{2} \eta_{\mu \nu} \eta^{\rho \sigma} R_{\rho \sigma}^{(2)}
\end{aligned}
$$


$g=\operatorname{det} g_{\mu \nu}$ and $R_{\rho \sigma}^{(1)}, R_{\rho \sigma}^{(2)}$ are first and second order of Riemann tensor for Weinberg definition (whose form can be seen from his textbook). Now that the energymomentum "tensors" are well classified above. We are now in the position to calculate the energy density of gravitational field created by a significant source. Here we will use various energy-momentum pseudo-tensors to calculate energy density of static Schwarzschild gravitational field in two kinds of gauge for comparison. When casting metric tensor solutions Eq. (16) and Eq. (18) into Eqs. (22-24), we can get the final results of energy density $t_{00}$ in the following table:

TABLE I. ENERGY DENSITY IN DIFFERENT GAUGE CONDTIONS

\begin{tabular}{|c|c|c|}
\hline $\begin{array}{c}\text { ENERGY } \\
\text { DENSITY }\end{array}$ & $\begin{array}{c}\text { GENERAL TRANSVERSE GAGUE } \\
\text { CONDITION }\end{array}$ & $\begin{array}{c}\text { HARMONIC GAUGE } \\
\text { CONDITION }\end{array}$ \\
\hline EINSTEIN & $\frac{(b-1)(5 b+3)}{(1+b)^{2}} \frac{G^{2} M^{2}}{r^{4}}$ & $\frac{G^{2} M^{2}}{2 r^{4}}$ \\
\hline $\begin{array}{c}\text { LANDAU- } \\
\text { LIFSHITZ }\end{array}$ & $\frac{13 b^{2}+30 b+21}{(1+b)^{2}} \frac{G^{2} M^{2}}{r^{4}}$ & $-\frac{7 G^{2} M^{2}}{2 r^{4}}$ \\
\hline WEINBERG & $\frac{21 b^{2}+30 b+13}{(1+b)^{2}} \frac{G^{2} M^{2}}{r^{4}}$ & $-\frac{3 G^{2} M^{2}}{2 r^{4}}$ \\
\hline
\end{tabular}

TABLE I tells us something interesting. (I) LandauLifshitz and Weinberg energy densities are non-positive in both kinds of gauge, even $b$ can be chosen any real number except $b=-1$. (II) Einstein energy density, although it is positive energy in harmonic gauge, meets a little complicated situation in radiation gauge. If $b$ is in the range of $-\frac{3}{5}<b<1$, Einstein's definition can lead to positive energy density of gravitational field as shown in Fig .1.
Fig .1 shows that that only Einstein's energy-momentum pseudo-tensor is a proper definition to calculate energy density of pure gravitational field in some cases. This is a rather interesting conclusion never been reported before, and perhaps bring some new insight about the static Schwarzschild space time (even black hole)[9].

\section{SUMMARY}

In this paper, firstly, we prove that Eq. (1) is a general transverse gauge condition in general relativity for linear approximation. This gauge condition can play the same role as the radiation gauge condition in electrodynamics. Then, by using Eq. (1), the energy density of gravitational has been revisited. we proved that the energy distribution of pure gravitational field out of the source can be upgraded by using the Einstein, Weinberg and LandauLifshitz energy-momentum pseudo-tensors in general transverse gauge. Finally, by the concrete computing the energy density of static Schwarzschild solution, we find the expression for Einstein is the most useful definition of energy-momentum in calculating energy density of gravity. This result perhaps bring some new physical effects in universe [10].

Finally, we remark that we have restricted our discussion to the weak-field approximation in this paper. Furthermore, we only calculate the pure energy density of the simplest static Schwarzschild gravitational field. for the application to other black-hole is also a non-trivial work and leave to future studies.

\section{ACKNOWLEDGMENT}

This work is supported by the 2014 Hubei Education Department Scientific Project No. B2014058, and 2013 Hubei university of medicine graduate start-up fund N0. 2013QDJZR03.

\section{REFERENCES}

[1] S.Weinberg,The quantum theory of fields. Vol.I.(Cambridge University ,2005).Chapter 8. P345 -P347.

[2] C. W. Misner, K. S. Thorne, and J. A. Wheeler, Gravitation (Freeman, San Francisco, 2009)

[3] Luke M.Butcher,Phys.Rev.D 82,104040(2010).

[4] M. Sharif, Neelum Bashir, Effects of Electromagnetic Field on Energy Density Inhomogeneity in Self-Gravitating Fluids. Gen. Relativ. Gravit. 44(2012)1725-1737

[5] Anirudh Pradhan, Shyam Sundar Kumhar. Int. J. Math. Eng. Sci. (IJMES), 1:74-89, 2012

[6] Adam J. Christopherson, Gauge conditions in combined dark energy and dark matter systems, Phys.Rev.D82:083515,2010..

[7] Xiang-Song Chen, Ben-Chao Zhu, Tensor gauge condition and tensor field decomposition. (2013), unprint.

[8] Changjun Gao, Phys. Rev. D 85, 023533(2012).

[9] László B. Szabados. Mass, gauge conditions and spectral properties of the Sen-Witten and 3-surface twistor operators in closed universes, Class. Quantum Grav (2014), soon printed:

[10] Zachariah B. Etienne, John G. Baker, Improved Moving Puncture Gauge Conditions for Compact Binary Evolutions ,Phys. Rev. D 90, 064032 (2014)

Figure 1. The range of parameter $b$ 